\title{
La construcción ambiental del bienestar urbano. Caso de Medellín, Colombia
}

\author{
Peter Charles Brand *
}

\begin{abstract}
The approach of this paper, more than being addressed to 'objective' environmental problems -the state of ecosystems and natural resources-, examines the emerging conditions of the environmental problem and the construction within this issue of a set of ideas, arguments and intervention objects to handle the cities. That analytical perspective requires to dialectically relate the urban environmental proposals with the general urban development dynamics, as well as with the general contemporary contradictions and conflicts within cities and regions. The paper takes as a reference the state reforms and the dismantling of public welfare policies and institutions. Having this as a starting point, the transference of the welfare idea to environmental issues is explored, as well as the symbolic construction of this idea in the cities through the planning discourse and the aesthetic representations of urban space, both moved by specialized institutions.
\end{abstract}

Keywords: environment, sustainable city, metaphor, welfare discourse, urban shape.

\section{Resumen}

El enfoque adoptado en este trabajo no se dirige a los problemas ambientales 'objetivos' -el estado de los ecosistemas y los recursos naturales-, sino más bien se escudriñan las condiciones de surgimiento de la problemática ambiental y la construcción en ella de un conjunto de ideas, argumentos y objetos de intervención para manejar las ciudades. Dicha perspectiva analítica exige intentar relacionar dialécticamente las propuestas ambientales urbanas con la dinámica general del desarrollo urbano y las contradicciones contemporáneas al interior de las ciudades y las regiones. El trabajo toma como referencia las reformas del Estado y el desmonte de las políticas e instituciones públicas de bienestar. A partir de ahí se explora la transferencia de la idea de bienestar hacia el medio ambiente y la construcción simbólica de esta idea en las ciudades, mediante el discurso planificador y la representación estética en el espacio urbano, movilizados ambos por instituciones especializadas.

Palabras clave: medio ambiente, ciudad sostenible, metáfora, bienestar, discurso, forma urbana.

*Universidad Nacional de Colombia, campus Medellín. Correo-e: pbrand@epm.net.co 


\section{Introducción ${ }^{1}$}

El fenómeno de la globalización ha traído consigo no sólo la integración de la economía a escala mundial, sino también la diseminación ampliada de los discursos (como este) y de las ideas urbanísticas y territoriales sin precedentes en la historia (como las que se expondrán críticamente en este ensayo). No obstante, la reconfiguración de la relación global-local implícita en este proceso y la resignificación de lo local como plataforma de integración e identidad, la hegemonía neoliberal tiende a asegurar un cerramiento tenaz de la comprensión de los desafíos contemporáneos del desarrollo, de manera que el surgimiento de las localidades esté cuidadosamente condicionado en su contenido y alcance.

En este trabajo se privilegia el discurso global del desarrollo sostenible y las propuestas urbanísticas locales que surgen de éste en términos de la ciudad sostenible. Evidentemente, se trata de un problema contemporáneo que se distingue por sus dimensiones planetarias, cuyas manifestaciones y forma de comprensión actual no hubieran sido posibles sin la globalización de la economía y todo lo que ésta significa en cuanto a los procesos de producción y distribución, los patrones de consumo, el uso de tecnologías, etcétera. A su vez, la creciente urbanización de la población en el ámbito mundial y la concentración de los procesos de producción y consumo en las ciudades -y con esto el redespliegue espacial de una parte importante de la fuente de los problemas ecológicos globales- conducía, dentro de este replanteamiento discursivo de los problemas del desarrollo, a la formulación de nuevas ideas sobre cómo deben ser las ciudades.

En efecto, bajo la sombra de una crisis ecológica global, las ciudades se reconceptualizaron desde una perspectiva ecológica. Hoy en día existe una vasta literatura y numerosas redes internacionales sobre la ciudad sostenible. El Informe Brundtland, Nuestro Futuro Común (1987) había dirigido por primera vez la atención ambiental hacia las ciudades, se compiló una agenda ambiental urbana en la Cumbre de Río de Janeiro en 1992, y en la Cumbre de Ciudades en Estambul (1996) se pudo empezar a demostrar 'prácticas ejemplares'. Al comenzar el siglo XXI, la sos-

${ }^{1}$ El presente trabajo está basado en el texto: "The sustainable city as metaphor: urban environmentalism in Medellín, Colombia", publicado en Mike Jenks y Rod Burgess (2000), Compact City. Sustainable Urban Forms for Developing Countries, Londres, Spon. 
tenibilidad es un objetivo universal para la orientación del desarrollo urbano, no sólo una preocupación 'de lujo' en los países desarrollados (ver, por ejemplo, CEC, 1990), sino algo que también encarrila las propuestas urbanas en el mundo entero (ver, por ejemplo, Jenks y Burgess, 2000).

Sin embargo, el enfoque adoptado aquí no se dirige a los problemas ambientales 'objetivos' -el estado de los ecosistemas y recursos naturales, y la modificación de ellos en el proceso de urbanización-, sino más bien se pretende escudriñar las condiciones de surgimiento de la problemática ambiental y la construcción en ella de un conjunto de ideas, argumentos y objetos de intervención para manejar las ciudades. Se rechaza la noción idealista de la revelación de valores inherentes en la naturaleza, para mirar el proceso de asignación social de significados y sentidos al medio ambiente. Esta perspectiva analítica -de la construcción simbólica de la naturaleza/medio ambiente- exige intentar relacionar dialécticamente las propuestas ambientales urbanas con la dinámica general del desarrollo urbano y las contradicciones contemporáneas al interior de las ciudades y regiones. Los enfoques ambientalistas ortodoxos tienden a ver estas contradicciones únicamente en términos de los límites ecológicos al desarrollo; ignoran por completo las contradicciones al interior de la organización social y, por lo tanto, la posibilidad de percibir en lo ambiental no solamente un problema ecológico, sino también una respuesta a otros problemas de orden social, tales como la cohesión, la gobernabilidad, la justicia, la representación política y estética, etcétera.

En esta dirección, el presente trabajo tomará como punto de partida o presupuesto las reformas del Estado y el desmonte de las políticas e instituciones públicas de bienestar. A partir de ahí se explorará la transferencia de la idea de bienestar hacia el medio ambiente y la construcción simbólica de esta idea en las ciudades, mediante el discurso planificador y la representación estética, movilizados ambos por instituciones especializadas. En la primera parte se esbozan algunas maneras generales de entender la ambientalización de la planeación urbana; en la segunda, se explora la calidad metafórica de las propuestas ambientales urbanas resumidas en términos como la 'ciudad sostenible' o 'ciudad compacta'; y en la tercera parte se ilustra la movilización de los nuevos sentidos en el caso de la ciudad de Medellín, Colombia. 


\section{Consideraciones teóricas sobre la problemática ambiental}

Esquemáticamente, podrían plantearse tres maneras de entender la ambientalización de las preocupaciones urbanas y de la planeación de las ciudades. El primero, y de hecho el sentido principal de los esfuerzos oficialistas, se basa en la objetivización del medio ambiente y la medición cuantitativa del estado de los recursos naturales. Se parte de la proposición de que el medio ambiente consiste en un complejo conjunto de sistemas ecológicos y recursos naturales, cuyo deterioro amenaza el soporte natural de la vida humana y la base del sistema de producción económica. Por lo tanto, cuidar el medio ambiente, administrarlo con prudencia y criterio ecológico se vuelve un principio (el de la sostenibilidad) que ha de orientar todas las actividades sociales. Y en un mundo urbanizado, las ciudades se convierten en centros clave para el desarrollo sostenible (UN-Habitat, 1996).

Dicha objetivización del medio ambiente conduce a lo que Hajer (1996) describe como la 'modernización ecológica'. Ésta consiste en la aplicación de la racionalidad técnico-científica para describir y explicar los problemas ambientales cuya resolución se adjudica, a su vez, a una alianza entre las grandes corporaciones científicas, industriales y gubernamentales. Esta alianza fija la agenda ambiental a escala global y define las pautas generales para acciones más locales y participativas. La estrategia está dominada por los centros de poder/conocimiento y la regulación del medio ambiente, que funciona con base en instrumentos tanto del mercado como de control normativo, y más recientemente con el involucramiento/cooptación de las organizaciones sociales (Harvey, 1996). Sin embargo, la tecnología y las técnicas siguen siendo los ejes. En estas últimas, como la contabilidad y la auditoría ambientales, los estudios de impacto ambiental, el monitoreo y los indicadores, reside la función de demostrar la eficacia de la estrategia de modernización ecológica y/o la necesidad de trabajarla con más determinación. En cuanto a resultados, las condiciones objetivas del medio ambiente, global, nacional y urbanísticamente, siguen en declive (ver PNUMA, 1999; Low et al., 2000).

Una segunda línea de interpretación, estrechamente articulada con la primera, postula en la ambientalización de la planeación la posibilidad de ampliar la participación ciudadana y fortalecer la democracia local. Comparte con la modernización ecológica la importancia del medio ambiente en cuanto a la cali- 
dad de vida: después de todo, ¿quién puede negar que el deterioro del medio ambiente -la inseguridad de los hogares producto de la amenaza de desastres naturales, los riesgos para la salud debido a la contaminación del aire y del agua, el empobrecimiento espiritual producto de la degradación estética del paisaje, etcétera- son asuntos de peso? Sin embargo, rechaza la determinación técnico-científica de las condiciones ambientales y la calidad de vida, y en su lugar reclama la necesidad de ampliar las perspectivas mediante el diálogo y el debate. En otras palabras, el asunto se politiza, y esta politización se produce tanto en el plano discursivo, para dotar a la noción de desarrollo sostenible de un sentido social concreto (Escobar, 1999; Acselrad, 1999), como en las prácticas concretas de administración del espacio.

Esta concepción ha sido importante en los asuntos urbanoregionales, ya que se trata de un acercamiento experiencial al medio ambiente en espacios o territorios concretos. Esta deja de ser una preocupación distante y abstracta, para entrometerse en la vida mundana y condicionar la sensibilidad individual y social hacia el entendimiento de las condiciones de existencia urbanas y regionales (Brand, 1996c; 1999). En consecuencia, el medio ambiente adquiere un sentido práctico-político accesible a los ciudadanos no expertos en el tema, y muchas veces en contradicción con el entendimiento técnico-científico de las autoridades ambientales y de planificación. De esta manera, el medio ambiente anima la cuestión de la política urbana, erigiéndose en un asunto que permite controvertir el devenir de las ciudades y de proyectos específicos de desarrollo. A partir de esta movilización general alrededor del medio ambiente, se cifran las esperanzas de nuevas dinámicas progresistas para 'dignificar' la existencia de las mayorías en los sectores degradados de las ciudades. En Colombia, por ejemplo, Viviescas (1993: 76) ha conceptuado:

En este punto se evidencia la potencia de la dimensión ambiental para darle fundamentación y coherencia a la configuración de la sociedad civil colombiana [...]: en primer lugar, porque la dota con marcos de referencia tangibles e inmediatos de consideración de la calidad de la existencia individual y colectiva que están más allá de los niveles 'básicos' o 'mínimos', superando el marco de la mera supervivencia y elevando con ello el horizonte de la reivindicación social y, en segundo lugar, porque le da a su accionar una significación que avanza sobre los aspectos meramente físicos y locales, dotándola de referentes e imaginarios que tienden a cualificar y ampliar la perspectiva política y cultural. 
Más allá de un medio experiencial y discursivo para redefinir políticamente el futuro de las ciudades, sobre lo cual hay evidencias alentadoras, el medio ambiente también se perfila, en su condición de medida de la calidad de vida urbano-regional, como nueva esfera de acción estatal a favor del bienestar colectivo. En Colombia, Palacio (1994: 26), por ejemplo, opina que “... lo ambiental, considerado como política social, bien podría ser el relevo de las políticas sociales de viejo cuño fundadas en el Estado Providencia". Ciertamente, el fuerte redireccionamiento de las instituciones del Estado hacia el medio ambiente presta cierta credibilidad a tal apreciación. Sin embargo, los efectos logrados hasta ahora, vistos a la luz del movimiento general de las condiciones de vida de las mayorías populares, advierte de la enorme distancia a recorrer todavía.

Una tercera opción de interpretación desarrolla las sugerencias respecto al significado político del medio ambiente antes señalado, pero ubica al medio ambiente, y los esfuerzos políticoinstitucionales sobre éste, dentro del conjunto de las transformaciones sociales. Se trata de una perspectiva teórica que rechaza la cosificación del medio ambiente como objeto y lo considera como una construcción social. De ahí surge el desafío de entenderlo no sólo como una cosa (ecosistema o recurso) para ser administrada, ni únicamente como algo en disputa política, sino más bien para examinar las condiciones mismas del surgimiento del medio ambiente como problemática. Esto conduce a aproximaciones históricas y antropológicas que obligan a acercarse al medio ambiente en su materialidad y contenido simbólico. Aunque abre perspectivas de reflexión filosófica, este enfoque lo sitúa sobre todo en la esfera de la praxis. Como explica Harvey (1996), todo proyecto sobre el medio ambiente es necesaria y simultáneamente un proyecto de cambio social.

En relación con las ciudades y su planeación, se plantea la necesidad de ubicar lo que se examinará en este trabajo -los discursos, las instituciones y las prácticas espaciales del ambientalismo urbano- en relación dialéctica con las transformaciones de orden económico y político, junto con las conciencias sociales que de ahí se deriven; es decir, intentar captar la plena significación del auge ambiental en relación con el redireccionamiento general de la administración urbana en las condiciones contemporáneas de modernización.

En términos más concretos, esta perspectiva permite ahondar en el análisis del significado de la reconstitución de la idea 
del bienestar en y a través del medio ambiente, examinando dichas posibilidades en relación con el desmonte de los propósitos, instituciones y programas tradicionales del Estado de bienestar (vivienda para todos, pleno empleo, seguridad social universal), el movimiento general hacia la privatización de la vida, y el reto de reconstruir un sentido de interés colectivo en un periodo de fragmentación e intensificación de desigualdades sociales (Brand, 1996a). Urbanísticamente, se abre el camino hacia el examen crítico del significado de una nueva idealización de la forma y la organización urbanas (la ciudad 'sostenible'). Asimismo, abordar las contradicciones y conflictos inherentes al medio ambiente abre nuevos debates relacionados con los derechos y deberes ciudadanos, la justicia social, la legitimación del Estado, el control social y la formación de un nuevo campo de relaciones disciplinarias (Dickens, 1996; Macnaughten y Urry, 1998). En fin, una constelación heterogénea y contradictoria de connotaciones que ofrece nuevos caminos de reflexión para entender y orientar el desarrollo urbano.

A continuación se pretende un acercamiento a esta amplitud de posibilidades mediante la comprensión de la 'ciudad sostenible' como metáfora. Se enfocará la ciudad sostenible no como una agenda técnica, sino como un ideal: una fijación de aspiraciones sociales y significados espaciales, con la capacidad de reorientar el sentido del desarrollo urbano y legitimar las acciones estatales en nombre del bien colectivo. De este modo, la medición de la sostenibilidad no se preocupa por los índices de consumo de energía, contaminación, riesgo o biodiversidad, y ni siquiera por la calidad de vida o la justicia urbana, sino por la manera y grado en que la idea adquiere el reconocimiento y apoyo públicos, para así contribuir a la cohesión social y la gobernabilidad urbana. Se busca captar la idea de la ciudad sostenible en su pureza metafórica: una figura para comunicar sentido y replantear los problemas socioespaciales de tal manera que sean políticamente manejables. En consecuencia, se deja atrás el análisis técnico de los sistemas de recursos naturales para concentrarse en el mundo de los símbolos y la movilización de significados a través del discurso planificador y la forma urbana.

La exploración de estas ideas en el caso de Medellín es apropiada en varios sentidos. La ciudad asimiló enérgicamente las propuestas del desarrollo sostenible a mediados de los años 80 , y en plena crisis social. La ciudad estaba sufriendo un agudo conflicto social asociado con los cárteles del narcotráfico, y lue- 
go se agudizaron la violencia política, los problemas de orden público y los índices de criminalidad, estos últimos más típicos de la ciudad latinoamericana. La urgente necesidad de reconstruir el sentido de unidad y orden en Medellín hizo que se explotara al máximo (y sin premeditación) el potencial ambiental, para enfrentar y encauzar los problemas socioespaciales.

\section{La metáfora de la 'ciudad sostenible'}

No obstante la reciente aparición de los inicios de una discusión crítica en torno a la noción de la sostenibilidad urbana (Dragsbeck Schmid, 1998; Parnwell y Turner, 1998; Marcuse, 1998), generalmente se ha dado una interpretación bastante literal a los términos 'desarrollo urbano sostenible' y 'ciudad compacta'. Sobre la base de una mirada 'objetiva' a las condiciones internas de las ciudades y su contribución a los problemas ambientales globales, se ha formulado una agenda ambiental urbana de múltiples dimensiones, pero que puede organizarse alrededor de los temas de: el consumo de energía, la polución, el transporte, la naturaleza, los estilos de vida y las formas de administración de lo urbano (Brand, 1996b). El reto de la ciudad sostenible se ha entendido con un sentido esencialmente técnico, que consiste en un esfuerzo sistemático de reducir o eliminar los problemas objetivamente definidos, cuantificables y medibles. Esto, a su vez, ha influido en la definición de una agenda socioespacial para la administración ambiental del desarrollo urbano. El enfoque ha sido pragmático e incremental: hacer lo posible en cada lugar particular y medir los resultados mediante un conjunto cada vez más amplio de indicadores de sostenibilidad.

La idea de la ciudad compacta, con su énfasis en la forma urbana, refleja esta tendencia general. Es menester observar que el debate no se ha limitado a una escala espacial específica ni se ha restringido exclusivamente a la ciudad como la unidad espacial definitiva. El enfoque pragmático e incremental ha permitido que, por un lado se destaque el edificio y el barrio, al tiempo que el reconocimiento de los sistemas urbanos lleve la atención a los sistemas regionales de asentamientos. La preocupación principal parece no ser tanto la compactación geográfica, sino más bien el impacto ambiental de las formas espaciales y los patrones de actividades. De esta manera, la idea de la ciudad compacta se revela tanto en su sentido literal como en el figurativo o como aspiración social. También podría plantearse, a la manera de Cas- 
tells (1996), que la explosión del espacio económico y social ha provocado esta respuesta, que intenta contrarrestar las consecuencias de la globalización y anclar localmente la experiencia del espacio mediante la referenciación ambiental en la producción de lugares.

Vista la sostenibilidad urbana desde esta perspectiva -el control sobre el espacio y la administración del lugar- las inconsistencias lógicas del desarrollo sostenible y las persistentes limitaciones de las prácticas urbanas dejan de ser problemáticas en el sentido ortodoxo. El desafío de la ciudad sostenible se vuelve menos un fin ambiental para convertirse en un medio de administración socioespacial. En otras palabras, la ciudad sostenible se puede entender como un proyecto expresamente político, $\mathrm{cu}-$ yos propósitos y efectos consisten en el replanteamiento de los problemas urbanos y la predefinición de las metas socioespaciales. La ciudad compacta se manifiesta como un dispositivo metafórico para condensar tales metas, para encapsular un nuevo conjunto de significados sociales y para cautivar la imaginación intelectual y social en la administración del lugar.

\subsection{La naturaleza de la metáfora}

El empleo de metáforas en el urbanismo tiene una larga historia. Hacia finales del siglo XIX se empezó a hablar de la 'ciudad-jardín', el modernismo introdujo las 'calles en el aire' y más tarde 'aldeas urbanas', y en el periodo actual del posmodernismo han proliferado metáforas como la 'ciudad vivible', la 'ciudad educadora' y la 'ciudad saludable'. Mientras que el uso de metáforas visuales constituye una dimensión corriente de la arquitectura (Jencks, 1977), el uso de metáforas literarias en la planeación urbana es tal vez igualmente común, pero menos estudiado. Incluso, podría pensarse que la metáfora es aún más pertinente en el urbanismo como mecanismo para comunicar significado, teniendo en cuenta que en el urbanismo el énfasis pasa del deleite estético a la intención social.

Por otro lado, la manera en que se comunica a través de la metáfora es de suma importancia. La disciplina del urbanismo se fundamenta en las ciencias sociales y, hoy en día, cada vez más en las ciencias de la tierra. La formación de ideas sobre las ciudades, la legitimación social de ellas y las prácticas de planeación se erigen sobre la base del conocimiento experto y la explicación racional de las dinámicas urbanas. Sin embargo, a pesar de estos 
cimientos técnico-científicos, el urbanismo recurre continuamente a las estratagemas retóricas, y las metáforas constituyen una parte importante de ellas. En contraste con la explicación racional, la metáfora es directa, atrayente y sugestiva; apela a las emociones y motiva. La metáfora no es analítica en el sentido de desagregar problemas y descubrir relaciones causales; más bien condensa significados y simboliza aspiraciones.

El movimiento general de la noción de sostenibilidad en la última década, y especialmente desde la Cumbre de las Ciudades realizada en Estambul en 1996, tiende a prestar credibilidad a este entendimiento metafórico. Si bien en sus inicios se entendía el desarrollo urbano sostenible con referencia a las condiciones objetivas de los sistemas de recursos naturales, ahora está asociado con una amplia gama de facetas de lo urbano y cierta subjetividad social en cuanto a la calidad de vida, interdependencia, bienestar, inclusión y cohesión social. En otras palabras, se ha abandonado la designación racional-objetiva de un campo específico de problemas espaciales (aquello asociado con el espacio natural) para incorporar la interpretación amplia y cualitativa de dichos problemas, en la comprensión integral de la ciudad y la vida urbana.

En este sentido, la ciudad sostenible puede verse como una metáfora vital para la renovación de las aspiraciones socio-urbanas en el contexto de la individualización posmoderna y la privatización de la vida, al mismo tiempo que insistentemente evoca la necesidad de pensar el futuro en medio de un presente abrumador por la velocidad y envergadura de los cambios que contiene (Brand, 1999). Por su parte, la metáfora de la ciudad sostenible es aun más potente, aun más resonante en sus connotaciones con la sociedad de consumo postindustrial. Después de todo, es contemporánea con el disco compacto y se alimenta de la asociación de éste en cuanto a lo último en tecnología, estilo de vida y eficiencia digital. La ciudad compacta se opone, tácitamente, a lo obsolescente y despreciable del crecimiento descontrolado, sea de ciudades, tubos catódicos, cuerpos sobrepasados de kilos y estilos de vida mal enfocados.

La metáfora no conlleva ningún significado preciso; más que denotar, evoca. Su significado no está determinado por la metáfora en sí misma, sino que se establece en conjugación con el contexto social y las reverberaciones que se producen en circunstancias específicas. En consecuencia, en la utilización de una metáfora siempre tiene que existir una manipulación consciente 
del significado, y una metáfora social, como la ciudad sostenible o el desarrollo urbano sostenible, nunca pueden ser una meta objetiva, sino una gama de posibilidades abiertas cuyas prioridades son determinadas por las urgencias del presente. En la sección siguiente se esbozan este contexto y estas prioridades en el caso de la ciudad de Medellín.

\subsection{El caso de Medellín}

Medellín es una ciudad de dos millones de habitantes y el centro de un área metropolitana que incorpora otros nueve municipios y una población total de tres millones, aproximadamente. En 1930 no existía sino un pequeño asentamiento de unas cien mil personas, ubicado cómoda y pasiblemente en un valle fértil de la cordillera central de los Andes. Ahora es una ciudad bulliciosa e industrial que crece cada día más por las laderas fuertemente inclinadas, reventándose. Medellín se enorgullece de una de las mejores tasas de provisión de servicios públicos en América Latina (99\% de los hogares tienen electricidad, 98\% acueducto y alcantarillado, y $82 \%$ teléfono doméstico), además tradicionalmente ha tenido un fuerte liderazgo e identidad regionales (Alcaldía de Medellín, 1998).

Sin embargo, en la década de los 80 las cosas empezaron a deteriorarse. La lenta acumulación de problemas urbanos y una fuerte recesión industrial se juntaron para producir el estancamiento de la economía, la pauperización extendida de la población y una aguda segregación socioespacial. (Departamento Administrativo de Planeación Metropolitana, 1985). Tensiones sociales latentes salieron a la superficie y luego explotaron con el surgimiento de los cárteles de drogas (Jaramillo, 1996). Se asentaba una cultura de violencia en la ciudad, y el número de homicidios en el área metropolitana llegó a 6,644 en 1991 (más o menos 1 por cada 400 personas al año). La violencia urbana alcanzó proporciones epidémicas, y todavía hoy el homicidio es la causa principal de muerte entre los hombres de 15 a 40 años y la obsesión cotidiana de todos los grupos sociales (Jaramillo, 1995; Veeduría Plan de Desarrollo, 1997).

Frente a esta situación se diseñaron dos campos de acción para reestablecer el orden público y el control estatal. El primero, más obvio y reconocido, fue abiertamente político. Por primera vez en la historia urbana de Colombia se creó una Comisión Presidencial para enfrentar los problemas sociales críticos 
de Medellín, en especial aquellos relacionados con los altos niveles de criminalidad y violencia generados por los cárteles de narcotraficantes. La estrategia incluyó una política de negociación con las organizaciones ilegales de la ciudad (narcotraficantes, movimientos guerrilleros y de delincuencia común), y el establecimiento de pactos de no violencia entre los actores en conflicto por el control territorial de los sectores populares de la ciudad (Programa Presidencial para Medellín y su Área Metropolitana, 1992; Jaramillo et al., 1998).

El segundo campo de respuesta fue explícitamente espacial y dirigido hacia el mejoramiento de las condiciones de vida de las áreas más pobres de la ciudad. En contraste con el enfoque represivo y de negociación política con los actores armados, esta vez se enfocó en la vida cotidiana de la gente en el contexto de crecientes niveles de violencia, desorden y desesperación. Teniendo en cuenta los niveles excepcionalmente altos de provisión de servicios públicos y la calidad generalmente aceptable de las condiciones físicas de la vivienda, el mejoramiento urbano se presentó como un desafío esencialmente (pero no exclusivamente) cualitativo. A continuación se examinará cómo, en este contexto de aguda crisis social, el medio ambiente y la idea de sostenibilidad jugaron un papel protagónico en la reconstrucción simbólica de un sentido de unidad y propósito común.

Al privilegiar el medio ambiente no se está sugiriendo que éste en sí constituya un campo eficaz para reestablecer el orden social en la ciudad, ni que tenga cualidades innatas que determinen el bienestar socioespacial. Se parte más bien de una postura analítica que entiende la necesidad de la construcción social de significados ambientales, y que la potencia del medio ambiente y de la sosteniblilidad únicamente pueden concretarse y explotarse en relación con el conjunto de problemas urbanos.

\section{La metáfora de la sostenibilidad y la movilización del significado en Medellín}

El desafío de fondo del ambientalismo urbano en Medellín fue movilizar significados alrededor del valor de la vida, la armonía y la coexistencia pacífica en y a través de la naturaleza, y utilizar la construcción de estos valores como un marco de referencia para repensar el futuro de una ciudad nublada por la violencia. El problema ambiental de Medellín residió no tanto en las condiciones objetivas de los sistemas de recursos naturales, sino más 
bien en cómo insertar el medio ambiente en el despliegue de los recursos formales (económicos, regulatorios y discursivos) para el desarrollo urbano, de manera que proporcionara coherencia y sentido a la vida urbana colectiva y legitimara el ejercicio de la autoridad pública.

Un ejercicio de esta naturaleza tenía sus raíces también en la realidad física de la ciudad. Los desastres ambientales causados por inundaciones y desprendimientos de tierra aseguraron que el medio ambiente ya existía como una preocupación entre la ciudadanía. Sin embargo, para que la metáfora de la sostenibilidad pudiera adquirir una aceptación social más amplia, los valores asociados con el medio ambiente tenían que ser movilizados y materializados en las formas simbólicas mediante las cuales la gente llevaba a cabo su vida cotidiana. Las siguientes secciones demostrarán cómo el discurso y la forma espacial proporcionaban este significado simbólico, y cómo la creación de instituciones especializadas garantizaban su eficaz transmisión.

\subsection{El discurso de la planeación}

La primera vez que el medio ambiente recibió atención seria fue en el plan de desarrollo de 1986. Aún en esos inicios, el contexto social fue de suma importancia en cuanto a la manera de presentar el medio ambiente como un problema. En ese periodo, la recesión económica y la influencia del narcotráfico empezaban a sentirse en la ciudad, agudizando los problemas sociales, y la construcción del prestigioso proyecto Metro de transporte masivo, iniciado en 1984, estaba rodeada de escándalos de corrupción que pronto llevarían a la suspensión de la obra (Acevedo et al., 1993). En consecuencia, la credibilidad de la administración local se veía severamente debilitada, así como la confianza del público en las instituciones locales, y un sentido de vulnerabilidad invadía a la ciudad. El medio ambiente emergía como un vector de esta vulnerabilidad, y podía ser explotado para desviar la impotencia o complicidad del Estado local en relación con el declive generalizado y crítico de las condiciones urbanas. La siguiente cita (Departamento Administrativo de Planeación Metropolitana, 1986: 39) ilustra cómo se logró tal efecto:

Asunto primordial como el que más es el de la seguridad ciudadana sin cuya presencia cualquier panorama que se plantee estará nublado por la zozobra y la intranquilidad; esta seguridad deberá estar afianzada en las medidas que adopten las entidades de gobierno para la prevención del delito en sus orígenes y para la represión del mismo en todas sus 
formas y manifestaciones. Sin embargo, estériles serán los esfuerzos de las autoridades en este campo mientras ellos no vayan acompañadas de una clara voluntad ciudadana de participar decididamente en la lucha contra este flagelo; tal aporte deberá estar representado en la colaboración para la vigilancia del vasto espacio público y de la propiedad privada, en la denuncia del delincuente, y fundamentalmente en la modificación del comportamiento social que se ha visto degradado por la tolerancia y aún por la complacencia con que se miran algunas modalidades del delito. Fundamental será también el mejoramiento de las medidas encaminadas a prevenir las tragedias ocasionadas por deslizamientos e inundaciones en sectores de alto riesgo ya identificados de la ciudad, la intensificación de los operativos para acciones de emergencia en caso de presentarse tales fenómenos, la disminución de la accidentalidad y la protección de la vida y bienes de los ciudadanos ante el riesgo de incendios. En la medida en que la comunidad pueda recuperar la tranquilidad y logre convertirse en coautora de su propia seguridad, podrá a su vez dedicar más energías al progreso de la ciudad y podrá tener más confianza en su futuro.

Un año después, un deslizamiento de tierra en el barrio Villa Tina resultó en la pérdida de 300 viviendas y 500 vidas, empujando el medio ambiente, de manera trágica y contundente, al primer plano de la atención pública. La relación medio ambiente/vulnerabilidad fue consolidada en la conciencia pública y elaborada en el discurso planificador, como se ilustra en el espíritu post-Brundtland de la siguiente notificación del líder del consejo municipal pronunciada en la inauguración de un foro abierto sobre medio ambiente y desarrollo urbano (Concejo de Medellín, 1989):

Unidos a la violencia, al hambre y la descomposición social, el ciudadano del siglo XXI asiste al doloroso espectáculo de la extinción de su medio natural y de su propia vida en un futuro que si no inmediato, muchos han asimilado como irremediable [...] De este foro saldrán entonces prioridades de acción. Es necesario reforzar la acción del Estado mediante la educación y el civismo comunitario. Nos urgen las soluciones y ello no da espera.

Al llegar a los años 90, la asociación del medio ambiente con la calidad de vida y la habitabilidad de la ciudad había sido consolidada, junto con una agenda técnica basada en la prevención y atención de desastres, la contaminación del agua y el manejo del bosque urbano; sin embargo, los problemas sociales se intensificaban. Propuestas nacionales para una reforma de la Constitución incluían el tema extremadamente sensible de la extradición de nacionales involucrados en el tráfico internacional de drogas, asunto presionado por el gobierno de los Estados Unidos y rechazado ferozmente por los cárteles de drogas que se concentraban en Medellín. Los últimos empezaron una campaña de intimi- 
dación y terror, al tiempo que en las calles de la ciudad se llevó a cabo una guerra entre la policía y los narcotraficantes, en la cual los tiroteos, masacres y bombas se volvieron sucesos cotidianos (Jaramillo, 1996). Los programas presidenciales antes mencionados tuvieron algún éxito, y en el plan de desarrollo de 1993 se argumentó que la ciudad empezaba a salir de lo peor de la violencia y el derrame de sangre. Un sentido de alivio permeaba a la ciudad, así como una conciencia urgente de la necesidad de reestablecer la armonía social, reconstruir las estructuras sociales y restaurar la confianza de la ciudad en sí misma. En ese momento se introdujo, por primera vez, la idea del desarrollo sostenible como uno de los ocho principios generales de dicho plan. El significado atribuido al término (Concejo de Medellín, 1993: 97) resultó ser una adaptación de la definición de Brundtland al contexto de violencia propio de Medellín:

En Medellín se planificará desde el punto de vista humano y con sentido de responsabilidad con las generaciones futuras, utilizando el criterio de desarrollo sostenible, para que en la medida en que se atiendan las necesidades presentes, se busque subsanar las carencias del pasado y se generen condiciones que garanticen la vida en paz y prosperidad para el futuro de los medellinenses. [...] La ciudad se regirá por los principios de respeto por la vida humana y de todo otro tipo; el desarrollo integral de los medellinenses, y por ende de la ciudad, deberá darse en perfecta armonía con los demás seres humanos y con el ecosistema que nos rodea.

La Cumbre de Río de Janeiro en 1992 representó, entre otras cosas, el reconocimiento internacional del medio ambiente como un asunto integral del desarrollo urbano. Este hecho proporcionó una autoridad adicional importante al empleo dado al medio ambiente en Medellín, como un dispositivo para la reconstitución conceptual de un sentido de unidad y armonía en una sociedad urbana fraccionada por la confrontación violenta, y como mecanismo para solidificar el optimismo en el futuro. El medio ambiente se asoció íntimamente con las nociones de equidad, seguridad, coexistencia pacífica, racionalidad y armonía, o sea, todas esas calidades tan notoriamente ausentes del mundo de las relaciones sociales en la ciudad en ese entonces. Esta estrategia no escrita continúa todavía hoy en día. Más recientemente se ha consolidado la agenda técnica (en especial relacionada con la contaminación del aire y el manejo de desechos sólidos) y se ha dado un mayor énfasis a la educación ambiental. Sin embargo, el medio ambiente está todavía asociado y articulado discursivamente a la cohesión social y la calidad de vida. El lado duro de la 
vida urbana se discute en términos de la competencia con otras ciudades en una economía globalizada y despiadada, al tiempo que el medio ambiente se presenta como la arena privilegiada de la razón, la armonía y la vida sana.

\subsection{Las instituciones}

En la sección anterior se describió cómo los planes de desarrollo establecieron el perfil general del discurso ambiental urbano y señalaron el conjunto de significados atribuibles al medio ambiente en relación con la problemática general de Medellín. Sin embargo, este discurso formal sobre el desarrollo urbano tenía una circulación limitada, circunscrita en buena parte a un pequeño grupo de expertos técnicos y políticos. Se requerían nuevas instituciones para que este mensaje se transmitiera al público en general y para que el discurso se extendiera más allá del ámbito limitado de los ejercicios de participación pública. Sobre todo, se necesitaban instituciones con responsabilidades ambientales, capaces de llevar el discurso a la acción práctica y así enraizar el significado simbólico en la experiencia cotidiana de la realidad urbana.

En el caso de Medellín hay dos instituciones de especial importancia. La primera se llama el Instituto Mi Río, creado en 1992 con el propósito expreso de proteger el río Medellín que corre a lo largo de la ciudad. Esta función se amplió rápidamente para abarcar el manejo integral de toda la cuenca hídrica, que coincide casi exactamente con la jurisdicción del área metropolitana. Al ser un organismo tanto coordinador como ejecutor, la junta directiva estaba conformada por el alcalde de Medellín, el director ejecutivo y el jefe de planeación del área metropolitana, los directores general y técnico de las Empresas Públicas de Medellín (acueducto, alcantarillado, energía eléctrica y telefonía), y el director ejecutivo de la Cámara de Comercio; más tarde se añadió un representante de la comunidad.

El énfasis inicial del Instituto Mi Río consistió en el mejoramiento paisajístico del río Medellín en su paso por la zona central de la ciudad, pero pronto este enfoque se amplió para incorporar el manejo de las numerosas quebradas afluyentes que caen por las laderas del valle, dando prioridad a los sectores populares de la ciudad. En el lapso 1995-1996 el Instituto implementó unos 600 proyectos que comprendían obras de ingeniería civil, mantenimiento de cauces y planes de arborización y paisajismo, que en 
su conjunto implicaron el tratamiento de 1'688,000 $\mathrm{m}^{2}$ y la siembra de 90,000 árboles. (Instituto Mi Río, 1996). Más recientemente el Instituto ha fortalecido su programa de desarrollo social, y la mitad de su presupuesto de unos us\$ 7.5 millones está dedicada a la educación ambiental y la generación de empleo (Instituto Mi Río, 1999).

Aunque el control del Instituto es tradicionalmente político y su estructura administrativa vertical, se desarrolló una estrategia participativa vigorosa. El Instituto alcanzó un amplio cuerpo de organizaciones comunitarias a través de su trabajo directo en los barrios de la ciudad, con campañas, concursos, talleres y festivales. También llevó a cabo una ágil política de relaciones públicas, mediante publicaciones, materiales educativos y promocionales, videos y campañas masivas. En fin, el Instituto Mi Río se convirtió rápidamente en una entidad moderna, eficiente y de alto perfil en la ciudad, gracias a sus estrechas relaciones con la comunidad y los medios de comunicación. Sus obras han tenido un fuerte impacto visual y en general disfruta de un amplio reconocimiento en la ciudad. Sobre todo, fue capaz de demostrar la 'sostenibilidad' en acción.

La segunda innovación institucional no resultó de una iniciativa local, sino que nació de la política ambiental nacional. En 1993 se creó en Colombia el Ministerio del Medio Ambiente y la ampliación del sistema de Corporaciones Autónomas Regionales para cubrir toda la extensión del territorio nacional, con responsabilidades exclusivamente de protección y gestión ambiental. Como autoridades ambientales regionales, sus funciones incluyen la autorización ambiental de proyectos y planes municipales de desarrollo. Las corporaciones se financian principalmente a través de una sobretasa catastral, y en un tiempo corto han adquirido poder financiero y capacidad técnica significativos, de manera que ahora constituyen actores fuertes en el proceso de desarrollo. Una curiosidad del sistema es que las ciudades grandes como Medellín caen dentro de la jurisdicción de dos corporaciones ambientales, una para el área rural y otra para el área urbana.

El asunto a destacar en el contexto del presente trabajo es que las corporaciones regionales ejercen su autoridad a través de las normas legales y el conocimiento técnico. Mientras que el Instituto Mi Río moviliza el significado mediante la acción concreta y proyectos urbanos, las corporaciones actúan como árbitros en el caso de disputas alrededor de estos significados. A tra- 
vés de sus poderes legales, su base de conocimiento científico y sus competencias técnicas, las corporaciones controlan los límites discursivos del ambientalismo urbano y se aseguran que su contenido social quede dentro del ámbito institucional del Estado. Además, estas nuevas instituciones ambientales se crearon al mismo tiempo que se iban desmontando, reformando o privatizando las instituciones estatales relacionadas con la vivienda, la salud y la seguridad social; de esta manera se logró consolidar el alejamiento de la idea del bienestar de sus esferas tradicionales y su traslado al medio ambiente.

\subsection{La forma espacial}

El desarrollo urbano sostenible, entendido como la movilización del significado a través de la forma simbólica, conlleva a una agenda espacial bastante diferente de aquella normalmente asociada con la idea de la ciudad sostenible o compacta. La dimensión funcional de la organización espacial (asuntos como el consumo energético, la contaminación, la salud pública, la conservación etc.), cede el campo a una racionalidad alternativa de tipo social. Mientras que el objetivo general es todavía el mismo -la sostenibilidad- los medios para lograrlo se transfieren desde la esfera de la tecnología hacia el mundo de la estética. La movilización sistemática del significado se logra, no a través de la reorganización, que es objetivo de las actividades espaciales, sino mediante la articulación sistemática de eventos visualmente significativos en el paisaje urbano. En otras palabras, no obstante el hecho de que casi inevitablemente resultan mejoras ecológicas y ambientales en la ciudad, se produce implícitamente una estetización del ideal de la sostenibilidad urbana.

La experiencia en la ciudad de Medellín puede describirse en términos de tres aspectos principales, los cuales, debe notarse, son también características del ambientalismo urbano en general: la elevación de los componentes naturales del espacio urbano a un estatus clave entre el conjunto de problemas urbanos, el enverdecimiento de la forma urbana, y el redireccionamiento de eventos socialmente significativos al espacio 'natural'.

En Medellín, el primer aspecto se persiguió más claramente en relación con las quebradas afluyentes que descienden, en ocasiones ferozmente, de las laderas fuertemente inclinadas del valle principal. Estos accidentes geográficos habían actuado, tradicionalmente, como barreras entre los barrios o sectores de la 
ciudad y como basureros para el depósito de desechos domésticos. Además, los riesgos asociados con los crecientes de los caudales e inestabilidad de los taludes se había incrementado con la paulatina invasión y construcción de viviendas. Una vez que se cumplió el tratamiento paisajístico del río principal, el Instituto Mi Río dirigió sus esfuerzos hacia estas quebradas afluyentes y empezó a explotar las posibilidades estéticas del sistema hídrico para el mejoramiento de los sectores populares de la ciudad.

El segundo aspecto tiene que ver con el enverdecimiento (greening) urbano o el desplazamiento generalizado de la naturaleza dentro del espacio urbano. El árbol, en particular, simboliza y simplifica la idea de la 'naturaleza' y sus valores de paz y armonía. Desde luego esta tendencia de enverdecimiento puede racionalizarse con base en la función ecológica del bosque urbano con sus efectos de descontaminación del aire, control micro-climático, estabilización de suelos y conservación de hábitat para la fauna urbana. Sin embargo, en Medellín el 'alcalde verde' fue especialmente influyente en la promoción de árboles socialmente productivos (frutales) y el goce estético no utilitario del ambiente verde. Además, las posibilidades de enverdecimiento en un clima húmedo tropical fueron enormes, y a través del Instituto Mi Río y otras organizaciones se han sembrado aproximadamente 650,000 árboles en el espacio público en los últimos quince años (Departamento Administrativo de Planeación Metropolitana, 1999). En consecuencia, la apariencia de la ciudad ha cambiado radicalmente, con la concentración de los árboles en espacios de flujos (vías, cauces de ríos y quebradas) así como en parques y cualquier espacio no construido.

El tercer aspecto, tal vez el más radical e innovador en términos de la reorganización espacial, tiene que ver con la extensión (y quizás eventual desplazamiento) del espacio simbólico de la ciudad desde el centro histórico al río principal (Viviescas, 1998; Plan Estratégico, 1997). Este traslado implica una distancia geográfica de entre uno y cinco kilómetros, pero con unos obstáculos mucho mayores. El significado cultural del centro histórico es todavía bastante fuerte, no obstante la reciente reubicación del sector financiero a una localización intermedia y una preocupación amplia por el deterioro físico y social del centro tradicional. Más importantes aún son las dificultades físico-espaciales. Los alrededores del río Medellín presentan un ambiente agreste e inhóspito, pues el río fue canalizado en los años 50 y se convirtió en un 'corredor multimodal de transporte' donde se acomodaron 
el eje principal del sistema vial metropolitano, el ferrocarril y luego el Metro, líneas de transmisión de energía y ductos para el transporte de petróleo y gas: un verdadero monumento -por lo demás bastante eficiente- a la ingeniería civil y el modernismo urbanístico. A pesar de un programa de descontaminación del agua iniciado a comienzos de los años 80 , es un espacio con polución, ruidoso, altamente restringido por las obras de infraestructura y con muy pocos objetos arquitectónicos de algún valor. No obstante, desde la realización de trabajos paisajísticos entre 1993-1994, las autoridades locales han logrado transferir al corredor del río algunos festivales principales, como las celebraciones navideñas, han inventado con éxito numerosos eventos culturales y deportivos, y lo han convertido en ciclovía recreativa los domingos, cuando se llena de miles de ciclistas y patinadores.

Esta conversión del corredor del río - un espacio urbano dominado por una autopista- en el lugar principal de la ciudad, va en contra de toda lógica tradicional de la planeación y diseño urbanos. Sólo puede encontrase una explicación en la elevación y movilización de los valores ambientales a través del discurso y las instituciones ambientales, y la estetización del medio ambiente por encima de cualquier consideración de tipo funcional.

\section{Conclusiones}

Este trabajo se ha enfocado en el contenido simbólico del ambientalismo urbano, explorando la movilización de significados en el caso de Medellín. Es menester clarificar que ese contenido simbólico no está divorciado de la racionalidad técnica del manejo de los sistemas ambientales o de la modernización ecológica; simplemente opera en otro nivel. La racionalidad técnica consiste en una explicación de una realidad externa, mientras que la dimensión simbólica condensa e internaliza significados. En el proceso, los objetos de la agenda técnica de la ciudad sostenible sufren una especie de metamorfosis: se vuelven, por decirlo así, etéreos, representaciones de algo diferente a ellos mismos, objetos del deseo colectivo.

La ciudad de Medellín se presenta como una ilustración clara de la explotación sistemática del poder simbólico del medio ambiente para enfrentar los malestares urbanos. Si bien Medellín es un ejemplo exagerado del esfuerzo ambiental por contener la descomposición social, está lejos de constituir un caso único en cuanto a su problemática urbana general. Algo semejante está 
ocurriendo en todas las ciudades, como componente constitutivo de la reorganización socioespacial local en las condiciones de globalización. El análisis de Medellín demuestra que el significado tiene que ser permanentemente reconstruido de acuerdo con la evolución de las condiciones sociales y políticas. Está por verse todavía cómo esta explotación simbólica y relacional del medio ambiente se concretará en tiempos y en lugares distintos.

\section{Bibliografía}

Acevedo, J., J. Salazar y W. Case (1993), El metro de Medellín: una ilusión costeada por todos los colombianos, Fonade/ Instituto Ser, Bogotá.

Acselrad, Henri (1999), "Sustentabilidad y ciudad", EURE, vol. Xxv, núm. 74, pp. 35-46.

Alcaldía de Medellín (1998), Por una ciudad más humana: Plan de Desarrollo de Medellín 1998-200, Alcaldía de Medellín, Medellín.

Brand, Peter (1996a), "La modernización y sus sombras”, en Producción, uso y consumo de ciudades, Fundación Hábitat Colombia, Bogotá.

_- (1996b), "El perfil emergente de la ciudad sostenible", Anotaciones sobre planeación, (Universidad Nacional de Colombia, Sede Medellín), núm. 44, pp. 15-26.

_ (1996c), "Urban environmentalism: In the twilight between vice and virtue", Urban Design International, 1 (4), pp. 357-360.

- (1998), Environmentalism and the configuration of urban space: contemporary city development in Medellin, Colombia, (tesis de doctorado sin publicar), Oxford Brookes University, Joint Centre for Urban Design, Oxford.

_ (1999), "The environment and postmodern spatial consciousness: a sociology of urban environmental agendas", Journal of Environmental Planning and Management, 42 (5), pp. 631-648. 
Castells, Manuel (1996), The information age: economy, society and culture, Vol.1 The rise of the network society, Blackwell, Oxford.

Commission of the European Communities (1990), Green paper on the urban environment, European Commission, Bruselas.

Concejo de Medellín (1989), Problemática ambiental del Valle de Aburrá, Concejo de Medellín, Medellín.

- (1993), Plan general de desarrollo para Medellín, Concejo de Medellín, Medellín.

Departamento Administrativo de Planeación Metropolitana (1985), Plan de Desarrollo Metropolitano del Valle de Aburrá, Área Metropolitana del Valle de Aburrá, Medellín.

— (1986), Plan de Desarrollo de Medellín, Municipio de Medellín, Medellín.

- (1999), Plan de Ordenamiento Territorial, (Documento de soporte técnico vol. 1), Municipio de Medellín, Medellín.

Dickens, Peter (1996), Reconstructing nature: alienation, emancipation and the division of labour, Routledge, London.

Dragsbaek Schmid, J. (1998), "Globalisation and inequality in urban South-East Asia", Third World Planning Review, 20 (2), pp. 127-145.

Escobar, Arturo (1999), "El desarrollo sostenible: diálogo de discursos", en A. Escobar, El Final del Salvaje, Cerec/Instituto Colombiano de Antropología, Bogotá.

Hajer, Maarten (1996), The politics of environmental discourse: ecological modernization and the policy process, Oxford University Press, Oxford. 
Harvey, David (1996), Justice, nature and the geography of difference, Blackwell, Oxford.

Instituto Mi Río (1996), Balance de gestión 1995-1996, Instituto Mi Río, Medellín.

- (1999), El manejo integral de la cuenca del río Medellín, Instituto Mi Río, Medellín.

Jaramillo, Ana María (1995), Control social y criminalidad en el Medellín del siglo Xx, Desde la Región (Boletín núm. 19), Corporación Región, Medellín.

- (1996), No era culpa de Pablo Escobar: Medellín sigue entre la vida y la muerte, Desde la Región (Boletín núm. 21), Corporación Región, Medellín.

_- A. Ceballos y M. Villa (1998), En la encrucijada: conflicto y cultura política en el Medellín de los noventa, Corporación Región, Medellín.

Jencks, Charles (1977), The language of post-modern architecture, Academy, Londres.

Jenks, Mike y Rod Burgess (2000), Compact cities. Sustainable urban forms for developing countries, Spon, Londres.

Low, N., B. Gleeson, I. Elander y R. Lidskog (2000), Consuming cities: The urban environment in the global economy after the Rio Declaration, Routledge, Londres.

Macnaughten, Phil y John Urry (1998), Contested Natures, Sage, Londres.

Marcuse, Peter (1998), "Sustainability is not enough", Environment and Urbanization, 10 (2), pp. 103-111.

Palacio, Germán (1994), "Notas preliminares sobre la redefinición jurídica de las relaciones jurídicas con la naturaleza", en Derecho y Medio Ambiente II (memorias del II Simposio Internacional sobre Derecho Ambiental), Corporación Penca de Sábila, Medellín. 
Parnwell, M. y S. Turner (1998), "Sustaining the unsustainable? City and society in Indonesia", Third World Planning Review, 20 (2), pp. 147-163.

Plan Estratégico (1997), El futuro de la ciudad metropolitana, 2015, Alcaldía de Medellín, Medellín.

Programa Presidencial para Medellín y su Área Metropolitana (1992), Medellín, en el camino a la concertación: Informe de gestión 1990-1992, Presidencia de la República, Medellín.

PNUMA (1999), Global environmental outlook 2000, <http:// www.grida.no/geo2000>

uN-Hábitat (1996), Un mundo en proceso de urbanización: informe mundial sobre los asentamientos humanos, Tercer Mundo/Inurbe/FNA, Bogotá.

Veeduría Plan de Desarrollo (1997), Evaluación del Plan de Desarrollo de Medellin: la seguridad y la convivencia, Veeduría Plan de Desarrollo, Medellín.

Viviescas, Fernando (1993), "La calidad de la vivienda y de la ciudad”, Revista Camacol, núm. 57, Colombia, pp. 7389.

Viviescas, Fernando (1998), "El Plan estratégico y la imaginación de la ciudad: el caso del tren-bulevard Medellín", en Y. Campos e I. Ortiz (eds.), La ciudad observada: violencia, cultura y política, Tercer Mundo/Observatorio de Cultura Urbana, Bogotá.

Enviado: 31 de mayo de 2001 Aceptado: 27 de junio de 2001 